\title{
The multiplicative norm convergence in normed Riesz algebras
}

\author{
Abdullah Aydin (1) \\ Department of Mathematics, Mus Alparslan University, Muş, Turkey
}

\begin{abstract}
A net $\left(x_{\alpha}\right)_{\alpha \in A}$ in an $f$-algebra $E$ is called multiplicative order convergent to $x \in E$ if $\left|x_{\alpha}-x\right| \cdot u \stackrel{\mathrm{o}}{\rightarrow} 0$ for all $u \in E_{+}$. This convergence was introduced and studied on $f$-algebras with the order convergence. In this paper, we study a variation of this convergence for normed Riesz algebras with respect to the norm convergence. A net $\left(x_{\alpha}\right)_{\alpha \in A}$ in a normed Riesz algebra $E$ is said to be multiplicative norm convergent to $x \in E$ if $\left\|\left|x_{\alpha}-x\right| \cdot u\right\| \rightarrow 0$ for each $u \in E_{+}$. We study this concept and investigate its relationship with the other convergences, and also we introduce the $m n$-topology on normed Riesz algebras.
\end{abstract}

Mathematics Subject Classification (2020). 46A40, 46E30

Keywords. $m n$-convergence, normed Riesz algebra, $m n$-topology, Riesz spaces, Riesz algebra, mo-convergence

\section{Introduction and preliminaries}

Let us recall some notations and terminologies used in this paper. An ordered vector space $E$ is said to be vector lattice (or, Riesz space) if, for each pair of vectors $x, y \in E$, the supremum $x \vee y=\sup \{x, y\}$ and the infimum $x \wedge y=\inf \{x, y\}$ both exist in E. For $x \in E$, $x^{+}:=x \vee 0, x^{-}:=(-x) \vee 0$, and $|x|:=x \vee(-x)$ are called the positive part, the negative part, and the absolute value of $x$, respectively. A vector lattice $E$ is called order complete if every nonempty bounded above subset has a supremum (or, equivalently, whenever every nonempty bounded below subset has an infimum). A vector lattice is order complete if and only if $0 \leq x_{\alpha} \uparrow \leq x$ implies the existence of the $\sup x_{\alpha}$. A partially ordered set $A$ is called directed if, for each $a_{1}, a_{2} \in A$, there is another $a \in A$ such that $a \geq a_{1}$ and $a \geq a_{2}$ (or, equivalently, $a \leq a_{1}$ and $a \leq a_{2}$ ). A function from a directed set $A$ into a set $E$ is called a net in $E$. A net $\left(x_{\alpha}\right)_{\alpha \in A}$ in a vector lattice $E$ is order convergent (or o-convergent, for short) to $x \in E$, if there exists another net $\left(y_{\beta}\right)_{\beta \in B}$ satisfying $y_{\beta} \downarrow 0$, and for any $\beta \in B$ there exists $\alpha_{\beta} \in A$ such that $\left|x_{\alpha}-x\right| \leq y_{\beta}$ for all $\alpha \geq \alpha_{\beta}$. In this case, we write $x_{\alpha} \stackrel{o}{\rightarrow} x$. An operator $T: E \rightarrow F$ between two vector lattices is called order continuous whenever $x_{\alpha} \stackrel{\circ}{\rightarrow} 0$ in $E$ implies $T x_{\alpha} \stackrel{\circ}{\rightarrow} 0$ in $F$. A vector $e \geq 0$ in a vector lattice $E$ is said to be a weak order unit whenever the band generated by $e$ satisfies $B_{e}=E$, or equivalently, whenever for each $x \in E_{+}$we have $x \wedge n e \uparrow x$; see much more information of vector lattices for example $[1,2,16,17]$. Recall that a net $\left(x_{\alpha}\right)_{\alpha \in A}$ in a vector lattice $E$ is

Email address: aaydin.aabdullah@gmail.com

Received: 28.10.2019; Accepted: 17.04.2020 
unbounded order convergent (or shortly, uo-convergent) to $x \in E$ if $\left|x_{\alpha}-x\right| \wedge u \stackrel{o}{\rightarrow} 0$ for every $u \in E_{+}$. In this case, we write $x_{\alpha} \stackrel{u o}{\longrightarrow} x$, we refer the reader for an exposition on uo-convergence to [3,5-11].

A vector lattice $E$ under an associative multiplication is said to be a Riesz algebra (or, shortly, $l$-algebra) whenever the multiplication makes $E$ an algebra (with the usual properties), and besides, it satisfies the following property: $x \cdot y \in E_{+}$for every $x, y \in E_{+}$. A Riesz algebra $E$ is called commutative whenever $x \cdot y=y \cdot x$ for all $x, y \in E$. Also, a subset $A$ of an $l$-algebra $E$ is called $l$-subalgebre of $E$ whenever it is also an $l$-algebra under the multiplication operation in $E$

An $l$-algebra $X$ is called: d-algebra whenever $u \cdot(x \wedge y)=(u \cdot x) \wedge(u \cdot y)$ and $(x \wedge y) \cdot u=$ $(x \cdot u) \wedge(y \cdot u)$ holds for all $u, x, y \in X_{+}$; almost $f$-algebra if $x \wedge y=0$ implies $x \cdot y=0$ for all $x, y \in X_{+} ; f$-algebra if, for all $u, x, y \in X_{+}, x \wedge y=0$ implies $(u \cdot x) \wedge y=(x \cdot u) \wedge y=0$; semiprime whenever the only nilpotent element in $X$ is zero; unital if $X$ has a multiplicative unit. Moreover, any $f$-algebra is both $d$ - and almost $f$-algebra (cf. $[2,12,13,17]$ ). A vector lattice $E$ is called Archimedean whenever $\frac{1}{n} x \downarrow 0$ holds in $E$ for each $x \in E_{+}$. Every Archimedean $f$-algebra is commutative; see for example [13, p.7]. Assume $E$ is an Archimedean $f$-algebra with a multiplicative unit vector $e$. Then, by applying [17, Thm.142.1(v)], in view of $e=e \cdot e=e^{2} \geq 0$, it can be seen that $e$ is a positive vector. On the other hand, since $e \wedge x=0$ implies $x=x \wedge x=(x \cdot e) \wedge x=0$, it follows that $e$ is a weak order unit (cf.[12, Cor.1.10]). In this article, unless otherwise, all vector lattices are assumed to be real and Archimedean and all $l$-algebras are assumed to be commutative.

A net $\left(x_{\alpha}\right)_{\alpha \in A}$ in an $f$-algebra $E$ is called multiplicative order convergent (or shortly, mo-convergent) to $x \in E$ whenever $\left|x_{\alpha}-x\right| \cdot u \stackrel{\circ}{\rightarrow} 0$ for all $u \in E_{+}$. Also, it is called mo-Cauchy if the net $\left(x_{\alpha}-x_{\alpha^{\prime}}\right)_{\left(\alpha, \alpha^{\prime}\right) \in A \times A}$ mo-converges to zero. $E$ is called mo-complete if every mo-Cauchy net in $E$ is mo-convergent, and it is also called mo-continuous if $x_{\alpha} \stackrel{\text { o }}{\rightarrow} 0$ implies $x_{\alpha} \stackrel{\text { mo }}{\longrightarrow} 0$; see much more detail information [4]. Recall that a norm $\|\cdot\|$ on a vector lattice is said to be a lattice norm whenever $|x| \leq|y|$ implies $\|x\| \leq\|y\|$. A vector lattice equipped with a lattice norm is known as a normed Riesz space or normed vector lattice. Moreover, a normed complete vector lattice is called Banach lattice. A net $\left(x_{\alpha}\right)_{\alpha \in A}$ in a Banach lattice $E$ is unbounded norm convergent (or un-convergent) to $x \in E$ if $\left\|\left|x_{\alpha}-x\right| \wedge u\right\| \rightarrow 0$ for all $u \in E_{+}$(cf. [8-10,15]). We routinely use the following fact: $y \leq x$ implies $u \cdot y \leq u \cdot x$ for all positive elements $u$ in $l$-algebras. So, we can give the following notion.

Definition 1.1. An $l$-algebra $E$ which is at the same time a normed Riesz space is called a normed l-algebra whenever $\|x \cdot y\| \leq\|x\| .\|y\|$ holds for all $x, y \in E$.

Motivated by the above definitions, we give the following notion.

Definition 1.2. A net $\left(x_{\alpha}\right)_{\alpha \in A}$ in a normed $l$-algebra $E$ is said to be multiplicative norm convergent (or shortly, mn-convergent) to $x \in E$ if $\left\|\left|x_{\alpha}-x\right| \cdot u\right\| \rightarrow 0$ for all $u \in E_{+}$. Abbreviated as $x_{\alpha} \stackrel{\mathrm{mn}}{\longrightarrow} x$. If the condition holds only for sequences then it is called sequentially $m n$-convergence.

In this paper, we study only the $m n$ - cases because the sequential cases are analogous in general.

Remark 1.3. (i) For a net $\left(x_{\alpha}\right)_{\alpha \in A}$ in a normed $l$-algebra $E, x_{\alpha} \stackrel{\mathrm{mn}}{\longrightarrow} x$ implies $x_{\alpha}$. $y \stackrel{\mathrm{mn}}{\longrightarrow} x \cdot y$ for all $y \in E$ because of $\left\|\left|x_{\alpha} \cdot y-x \cdot y\right| \cdot u\right\| \leq\left\|\left|x_{\alpha}-x\right| \cdot|y| \cdot u\right\|$ for all $u \in E_{+} ;$see for example [12, p.1]. The converse holds true in normed $l$-algebras with the multiplication unit. Indeed, assume $x_{\alpha} \cdot y \stackrel{\mathrm{mn}}{\longrightarrow} x \cdot y$ for each $y \in E$. Fix $u \in E_{+}$. So, $\left\|\left|x_{\alpha}-x\right| \cdot u\right\|=\left\|\left|x_{\alpha} \cdot e-x \cdot e\right| \cdot u\right\| \stackrel{\mathrm{mn}}{\longrightarrow} 0$. 
(ii) In normed $l$-algebras, the norm convergence implies the $m n$-convergence. Indeed, by considering the inequality $\left\|\left|x_{\alpha}-x\right| \cdot u\right\| \leq\left\|x_{\alpha}-x\right\| \cdot\|u\|$ for any net $x_{\alpha} \stackrel{\mathrm{mn}}{\longrightarrow} x$, we can get the desired result.

(iii) If a net $\left(x_{\alpha}\right)_{\alpha \in A}$ is order Cauchy and $x_{\alpha} \stackrel{\mathrm{mn}}{\longrightarrow} x$ in a normed $l$-algebra then we have $x_{\alpha} \stackrel{\mathrm{mo}}{\longrightarrow} x$. Indeed, since the order Cauchy norm convergent net is order convergent to its norm limit, we can get the desired result.

(iv) In order continuous normed $l$-algebras, it is clear that the mo-convergence implies the $m n$-convergence.

(v) In order continuous normed $l$-algebras, following from the inequality $\left\|\left|x_{\alpha}-x\right| \cdot u\right\| \leq$ $\left\|x_{\alpha}-x\right\| .\|u\|$, the order convergence implies the $m n$-convergence.

(vi) In atomic and order continuous Banach lattice $l$-algebras, an order bounded and $m n$-convergent to zero sequence is sequentially mo-convergent to zero; see [9, Lem.5.1.].

(vii) For an $m n$-convergent to zero sequence $\left(x_{n}\right)$ in a Banach lattice $l$-algebra, there is a subsequence $\left(x_{n_{k}}\right)$ which sequentially mo-converges to zero; see [11, Lem.3.11.].

Example 1.4. Let $E$ be a Banach lattice. Fix an element $x \in E$. Then the principal ideal $I_{x}=\{y \in E: \exists \lambda>0$ with $|y| \leq \lambda x\}$, generated by $x$ in $E$ under the norm $\|\cdot\|_{\infty}$ which is defined by $\|y\|_{\infty}=\inf \{\lambda>0:|y| \leq \lambda x\}$, is an $A M$-space; see [2, Thm.4.21.].

Recall that a vector $e>0$ is called order unit whenever for each $x$ there exists some $\lambda>0$ with $|x| \leq \lambda e$ (cf. [1, p.20]). Thus, we have $\left(I_{x},\|\cdot\|_{\infty}\right)$ is $A M$-space with the unit $|x|$. Since every $A M$-space with the unit, besides being a Banach lattice, has also an $l$-algebra structure (cf. [2, p.259]). So, we can say that $\left(I_{x},\|\cdot\|_{\infty}\right)$ is a Banach lattice $l$-algebra. Therefore, for a net $\left(x_{\alpha}\right)_{\alpha \in A}$ in $I_{x}$ and $y \in I_{x}$, by applying [2, Cor.4.4.], we get $x_{\alpha} \stackrel{\mathrm{mn}}{\longrightarrow} y$ in the original norm of $E$ on $I_{x}$ if and only if $x_{\alpha} \stackrel{\mathrm{mn}}{\longrightarrow} y$ in the norm $\|\cdot\|_{\infty}$. In particular, take $x$ as the unit element $e$ of $E$. Then we have $E_{e}=E$. Thus, for a net $\left(x_{\alpha}\right)_{\alpha \in A}$ in $E$, we have $x_{\alpha} \stackrel{\mathrm{mn}}{\longrightarrow} y$ in the $\left(E,\|\cdot\|_{\infty}\right)$ if and only if $x_{\alpha} \stackrel{\mathrm{mn}}{\longrightarrow} y$ in the $(E,\|\cdot\|)$.

\section{The $m n$-convergence on normed $l$-algebras}

We begin the section with the next list of properties of $m n$-convergence which follows directly from the inequalities $|x-y| \leq\left|x-x_{\alpha}\right|+\left|x_{\alpha}-y\right|$ and ||$x_{\alpha}|-| x|| \leq\left|x_{\alpha}-x\right|$ for arbitrary net in $\left(x_{\alpha}\right)_{\alpha \in A}$ in vector lattice.

Lemma 2.1. Let $\left(x_{\alpha}\right)_{\alpha \in A}$ and $\left(y_{\beta}\right)_{\beta \in B}$ be two nets in a normed l-algebra $E$. Then the followings hold:

(i) $\quad x_{\alpha} \stackrel{\mathrm{mn}}{\longrightarrow} x \Longleftrightarrow\left(x_{\alpha}-x\right) \stackrel{\mathrm{mn}}{\longrightarrow} 0 \Longleftrightarrow\left|x_{\alpha}-x\right| \stackrel{\mathrm{mn}}{\longrightarrow} 0$;

(ii) if $x_{\alpha} \stackrel{\mathrm{mn}}{\longrightarrow} x$ then $y_{\beta} \stackrel{\mathrm{mn}}{\longrightarrow} x$ for each subnet $\left(y_{\beta}\right)$ of $\left(x_{\alpha}\right)$;

(iii) suppose $x_{\alpha} \stackrel{\mathrm{mn}}{\longrightarrow} x$ and $y_{\beta} \stackrel{\mathrm{mn}}{\longrightarrow} y$, then $a x_{\alpha}+b y_{\beta} \stackrel{\mathrm{mn}}{\longrightarrow} a x+$ by for any $a, b \in \mathbb{R}$;

(iv) if $x_{\alpha} \stackrel{\mathrm{mn}}{\longrightarrow} x$ then $\left|x_{\alpha}\right| \stackrel{\mathrm{mn}}{\longrightarrow}|x|$.

The lattice operations in normed $l$-algebras are $m n$-continuous in the following sense.

Proposition 2.2. Let $\left(x_{\alpha}\right)_{\alpha \in A}$ and $\left(y_{\beta}\right)_{\beta \in B}$ be two nets in a normed l-algebra E. If $x_{\alpha} \stackrel{\mathrm{mn}}{\longrightarrow} x$ and $y_{\beta} \stackrel{\mathrm{mn}}{\longrightarrow} y$ then $\left(x_{\alpha} \vee y_{\beta}\right)_{(\alpha, \beta) \in A \times B} \stackrel{\mathrm{mn}}{\longrightarrow} x \vee y$.

Proof. Assume $x_{\alpha} \stackrel{\mathrm{mn}}{\longrightarrow} x$ and $y_{\beta} \stackrel{\mathrm{mn}}{\longrightarrow} y$. Then, for a given $\varepsilon>0$, there exist indexes $\alpha_{0} \in A$ and $\beta_{0} \in B$ such that $\left\|\left|x_{\alpha}-x\right| \cdot u\right\| \leq \frac{1}{2} \varepsilon$ and $\left\|\left|y_{\beta}-y\right| \cdot u\right\| \leq \frac{1}{2} \varepsilon$ for every $u \in E_{+}$and for all $\alpha \geq \alpha_{0}$ and $\beta \geq \beta_{0}$. It follows from the inequality $|a \vee b-a \vee c| \leq|b-c|$ in vector lattices (cf. [2, Thm.1.9(2)]) that

$$
\begin{aligned}
\left\|\left|x_{\alpha} \vee y_{\beta}-x \vee y\right| \cdot u\right\| & \leq\left\|\left|x_{\alpha} \vee y_{\beta}-x_{\alpha} \vee y\right| \cdot u+\left|x_{\alpha} \vee y-x \vee y\right| \cdot u\right\| \\
& \leq\left\|\left|y_{\beta}-y\right| \cdot u\right\|+\left\|\left|x_{\alpha}-x\right| \cdot u\right\| \leq \frac{1}{2} \varepsilon+\frac{1}{2} \varepsilon=\varepsilon
\end{aligned}
$$


for all $\alpha \geq \alpha_{0}$ and $\beta \geq \beta_{0}$ and for every $u \in E_{+}$. That is, $\left(x_{\alpha} \vee y_{\beta}\right)_{(\alpha, \beta) \in A \times B} \stackrel{\mathrm{mn}}{\longrightarrow} x \vee y$.

The following proposition is similar to [4, Prop.2.7.], and so we omit its proof.

Proposition 2.3. Let $B$ be a projection band in a normed l-algebra $E$ and $P_{B}$ be the corresponding band projection. Then $x_{\alpha} \stackrel{\mathrm{mn}}{\longrightarrow} x$ in $E$ implies $P_{B}\left(x_{\alpha}\right) \stackrel{\mathrm{mn}}{\longrightarrow} P_{B}(x)$ in both $E$ and $B$.

A positive vector $e$ in a normed vector lattice $E$ is called quasi-interior point if and only if $\|x-x \wedge n e\| \rightarrow 0$ for each $x \in E_{+}$. If $\left(x_{\alpha}\right)$ is a net in a vector lattice with a weak unit $e$ then $x_{\alpha} \stackrel{\text { uo }}{\rightarrow} 0$ if and only if $\left|x_{\alpha}\right| \wedge e \stackrel{\text { o }}{\rightarrow} 0$; see [10, Lem. 3.5]. Also, there exist some results for the quasi-interior point case in [9, Lem. 2.11] and for p-unit case in [5, Thm. 3.2]. We give an expansion to normed $l$-algebras with the $m n$-convergence for quasi-interior points in the next result.

Proposition 2.4. Let $\left(x_{\alpha}\right)_{\alpha \in A}$ be a positive and decreasing net in a normed l-algebra $E$ with a quasi-interior point $e$. Then $x_{\alpha} \stackrel{\mathrm{mn}}{\longrightarrow} 0$ if and only if $\left(x_{\alpha} \cdot e\right)_{\alpha \in A}$ norm converges to zero.

Proof. The forward implication is immediate because of $e \in E_{+}$. For the converse implication, fix a positive vector $u \in E_{+}$and $\varepsilon>0$. Thus, for a fixed index $\alpha_{1}$, we have $x_{\alpha} \leq x_{\alpha_{1}}$ for all $\alpha \geq \alpha_{0}$ because of $\left(x_{\alpha}\right)_{\alpha \in A} \downarrow$. Then we have

$$
x_{\alpha} \cdot u \leq x_{\alpha} \cdot(u-u \wedge n e)+x_{\alpha} \cdot(u \wedge n e) \leq x_{\alpha_{1}} \cdot(u-u \wedge n e)+n\left(x_{\alpha} \cdot e\right)
$$

for all $\alpha \geq \alpha_{1}$ and each $n \in \mathbb{N}$. Hence, we get

$$
\left\|x_{\alpha} \cdot u\right\| \leq\left\|x_{\alpha_{1}}\right\| \cdot\|u-u \wedge n e\|+n\left\|x_{\alpha} \cdot e\right\|
$$

for every $\alpha \geq \alpha_{1}$ and each $n \in \mathbb{N}$. So, we can find $n$ such that $\|u-u \wedge n e\|<\frac{\varepsilon}{2\left\|x_{\alpha_{1}}\right\|}$ because $e$ is a quasi-interior point. On the other hand, it follows from $x_{\alpha} \cdot e \stackrel{\|\cdot\|_{1}}{\longrightarrow} 0$ that there exists an index $\alpha_{2}$ such that $\left\|x_{\alpha} \cdot e\right\|<\frac{\varepsilon}{2 n}$ whenever $\alpha \geq \alpha_{2}$. Since index set $A$ is directed, there exists another index $\alpha_{0} \in A$ such that $\alpha_{0} \geq \alpha_{1}$ and $\alpha_{0} \geq \alpha_{2}$. Therefore, we get

$$
\left\|x_{\alpha} \cdot u\right\|<\left\|x_{\alpha_{0}}\right\| \frac{\varepsilon}{2\left\|x_{\alpha_{0}}\right\|}+n \frac{\varepsilon}{2 n}=\varepsilon
$$

and so $\left\|x_{\alpha} \cdot u\right\| \rightarrow 0$.

Remark 2.5. A positive and decreasing net $\left(x_{\alpha}\right)_{\alpha \in A}$ in an order continuous Banach $l$ algebra $E$ with weak unit $e$ is $m n$-convergent to zero if and only if $x_{\alpha} \cdot e \stackrel{\|\cdot\|}{\longrightarrow} 0$. Indeed, it is known that $e$ is a weak unit if and only if $e$ is a quasi-interior point in an order continuous Banach lattice; see for example [1, p.135]. Thus, following from Proposition 2.4, one can get the desired result.

The $m n$-convergence passes obviously to any normed $l$-subalgebra $Y$ of a normed $l$ algebra $E$, i.e., for any net $\left(y_{\alpha}\right)_{\alpha \in A}$ in $Y$ with $y_{\alpha} \stackrel{\mathrm{mn}}{\longrightarrow} 0$ in $E$ implies $y_{\alpha} \stackrel{\mathrm{mn}}{\longrightarrow} 0$ in $Y$. For the converse, we give the following theorem whose proof is similar to [4, Thm. 2.10], and so we omit it.

Theorem 2.6. Let $Y$ be a normed l-subalgebra of a normed l-algebra $E$ and $\left(y_{\alpha}\right)_{\alpha \in A}$ be a net in $Y$. If $y_{\alpha} \stackrel{\mathrm{mn}}{\longrightarrow} 0$ in $Y$ then it mn-converges to zero in $E$ for both of the following cases hold;

(i) $Y$ is majorizing in $E$;

(ii) $Y$ is a projection band in $E$. 
It is known that every Archimedean vector lattice has a unique order completion; see [2, Thm. 2.24]. Moreover, Archimedean commutative l-algebra admits the unique extension multiplication to the order completion of it.

Theorem 2.7. Let $E$ and $E^{\delta}$ be order continuous normed l-algebras with $E^{\delta}$ being order completion of $E$. Then, for a sequence $\left(x_{n}\right)$ in $E$, the followings hold true:

(i) If $x_{n} \stackrel{\mathrm{mn}}{\longrightarrow} 0$ in $E$ then there is a subsequence $\left(x_{n_{k}}\right)$ of $\left(x_{n}\right)$ such that $x_{n_{k}} \stackrel{\mathrm{mn}}{\longrightarrow} 0$ in $E^{\delta}$

(ii) If $x_{n} \stackrel{\mathrm{mn}}{\longrightarrow} 0$ in $E^{\delta}$ then there is a subsequence $\left(x_{n_{k}}\right)$ of $\left(x_{n}\right)$ such that $x_{n_{k}} \stackrel{\mathrm{mn}}{\longrightarrow} 0$ in E.

Proof. Let $x_{n} \stackrel{\mathrm{mn}}{\longrightarrow} 0$ in $E$, i.e., $\left|x_{n}\right| \cdot u \stackrel{\|\cdot\|_{1}}{\longrightarrow} 0$ in $E$ for all $u \in E_{+}$. Now, let's fix $v \in E_{+}^{\delta}$. Then there exists $u_{v} \in E_{+}$such that $v \leq u_{v}$ because $E$ majorizes $E^{\delta}$. Since $\left|x_{n}\right| \cdot u_{v} \stackrel{\|\cdot\|_{1}}{\longrightarrow} 0$, by the standard fact in [1, Exer.13., p.25], there exists a subsequence $\left(x_{n_{k}}\right)$ of $\left(x_{n}\right)$ such that $\left(\left|x_{n_{k}}\right| \cdot u_{v}\right)$ order converges to zero in $E$. Thus, we get $\left|x_{n_{k}}\right| \cdot u_{v} \stackrel{\circ}{\rightarrow} 0$ in $E^{\delta}$; see [10, Cor.2.9.]. Then it follows from the inequality $\left|x_{n_{k}}\right| \cdot v \leq\left|x_{n_{k}}\right| \cdot u_{v}$ that we have $\left|x_{n_{k}}\right| \cdot v \stackrel{\mathrm{o}}{\rightarrow} 0$ in $E^{\delta}$. That is, $x_{n_{k}} \stackrel{\mathrm{mo}}{\longrightarrow} 0$ in the order completion $E^{\delta}$ because $v \in E_{+}^{\delta}$ is arbitrary. It follows from the order continuous norm that $x_{n_{k}} \stackrel{\mathrm{mn}}{\longrightarrow} 0$ in the order completion $E^{\delta}$.

For the converse, put $x_{n} \stackrel{\mathrm{mn}}{\longrightarrow} 0$ in $E^{\delta}$. Then, for all $u \in E_{+}^{\delta}$, we have $\left|x_{n}\right| \cdot u \stackrel{\|\cdot\|}{\longrightarrow} 0$ in $E^{\delta}$. In particular, for all $w \in E_{+},\left\|\left|x_{n}\right| \cdot w\right\| \rightarrow 0$ in $E^{\delta}$. Fix $w \in E_{+}$. Then, again by the standard fact in [1, Exer.13., p.25], we have a subsequence $\left(x_{n_{k}}\right)$ of $\left(x_{n}\right)$ such that $\left(x_{n_{k}}\right)$ is order convergent to zero in $E^{\delta}$. Thus, we get $\left|x_{n_{k}}\right| \cdot w \stackrel{\circ}{\rightarrow} 0$ in $E$. As a result, since $w$ is arbitrary, $x_{n_{k}} \stackrel{\mathrm{mo}}{\longrightarrow} 0$ in $E$. Therefore, one can get the result by using order continuous norm.

Recall that a subset $A$ in a normed lattice $(E, \mid \cdot \|)$ is said to almost order bounded if, for any $\epsilon>0$, there is $u_{\epsilon} \in E_{+}$such that $\left|\left(|x|-u_{\epsilon}\right)^{+}\|=\|\right| x\left|-u_{\epsilon} \wedge\right| x \mid \| \leq \epsilon$ for any $x \in A$. For a given normed $l$-algebra $E$, one can give the following definition: a subset $A$ of $E$ is called an l-almost order bounded if, for any $\epsilon>0$, there is $u_{\varepsilon} \in E_{+}$such that $\left\||x|-u_{\epsilon} \cdot|x|\right\| \leq \epsilon$ for any $x \in A$. Similar to [11, Prop.3.7.], we give the following work.

Proposition 2.8. Let $E$ be a normed l-algebra. If $\left(x_{\alpha}\right)_{\alpha \in A}$ is l-almost order bounded and $m n$-converges to $x$, then $\left(x_{\alpha}\right)_{\alpha \in A}$ converges to $x$ in norm.

Proof. Assume $\left(x_{\alpha}\right)_{\alpha \in A}$ is an $l$-almost order bounded net. Then the net $\left(\left|x_{\alpha}-x\right|\right)_{\alpha \in A}$ is also $l$-almost order bounded. For any fixed $\varepsilon>0$, there exists $u_{\varepsilon}>0$ such that

$$
|| x_{\alpha}-x\left|-u_{\epsilon} \cdot\right| x_{\alpha}-x|| \mid \leq \epsilon .
$$

Since $x_{\alpha} \stackrel{\mathrm{mn}}{\longrightarrow} x$, we have $\left\|\left|x_{\alpha}-x\right| \cdot u_{\varepsilon}\right\| \rightarrow 0$. Therefore, following from Proposition 2.2, we get $\left\|x_{\alpha}-x\right\| \leq \varepsilon$, i.e., $x_{\alpha} \rightarrow x$ in the norm.

Proposition 2.9. In an order continuous Banach l-algebra, every l-almost order bounded mo-Cauchy net converges $m n$ and in norm to the same limit.

Proof. Assume a net $\left(x_{\alpha}\right)_{\alpha \in A}$ is $l$-almost order bounded and mo-Cauchy in an order continuous Banach $l$-algebra $E$. Then the net $\left(x_{\alpha}-x_{\alpha^{\prime}}\right)_{\left(\alpha, \alpha^{\prime}\right) \in A \times A}$ is $l$-almost order bounded and is mo-convergent to zero. Thus, it $m n$-converges to zero by the order continuity of the norm. Hence, by applying Proposition 2.8, we get that the net $\left(x_{\alpha}-x_{\alpha^{\prime}}\right)_{\left(\alpha, \alpha^{\prime}\right) \in A \times A}$ converges to zero in the norm. It follows that the net $\left(x_{\alpha}\right)$ is norm Cauchy, and so it is norm convergent because $E$ is Banach lattice. As a result, we have that $\left(x_{\alpha}\right) m n$-converges to its norm limit by Remark 1.3(ii).

The multiplication in normed $l$-algebra is $m n$-continuous in the following sense. 
Theorem 2.10. Let $E$ be a normed l-algebra, and $\left(x_{\alpha}\right)_{\alpha \in A}$ and $\left(y_{\beta}\right)_{\beta \in B}$ be two nets in $E$. If $x_{\alpha} \stackrel{\mathrm{mn}}{\longrightarrow} x$ and $y_{\beta} \stackrel{\mathrm{mn}}{\longrightarrow} y$ for some $x, y \in E$ and each positive element of $E$ can be written as a multiplication of two positive elements then we have $x_{\alpha} \cdot y_{\beta} \stackrel{\mathrm{mn}}{\longrightarrow} x \cdot y$.

Proof. Assume $x_{\alpha} \stackrel{\mathrm{mn}}{\longrightarrow} x$ and $y_{\beta} \stackrel{\mathrm{mn}}{\longrightarrow} y$. Then $\left|x_{\alpha}-x\right| \cdot u \stackrel{\|\cdot\|}{\longrightarrow} 0$ and $\left|y_{\beta}-y\right| \cdot u \stackrel{\|\cdot\|}{\longrightarrow} 0$ for every $u \in E_{+}$. Let's fix $u \in E_{+}$and $\varepsilon>0$. So, there exist indexes $\alpha_{0}$ and $\beta_{0}$ such that $\left\|\left|x_{\alpha}-x\right| \cdot u\right\| \leq \varepsilon$ and $\left\|\left|y_{\beta}-y\right| \cdot u\right\| \leq \varepsilon$ for all $\alpha \geq \alpha_{0}$ and $\beta \geq \beta_{0}$.

Next, we show the $m n$-convergence of $\left(x_{\alpha} \cdot y_{\beta}\right)$ to $x \cdot y$. By considering the equality $|x \cdot y| \leq|x| \cdot|y|$ (cf. [12, p.1]), we have

$$
\begin{aligned}
\left\|\left|x_{\alpha} \cdot y_{\beta}-x \cdot y\right| u\right\| & =\left\|\left|x_{\alpha} \cdot y_{\beta}-x_{\alpha} \cdot y+x_{\alpha} \cdot y-x \cdot y\right| \cdot u\right\| \\
& \leq\left\|\left|x_{\alpha}\right| \cdot\left|y_{\beta}-y\right| \cdot u\right\|+\left\|\left|x_{\alpha}-x\right| \cdot|y| \cdot u\right\| \\
& \leq\left\|\left|x_{\alpha}-x\right| \cdot\left|y_{\beta}-y\right| \cdot u\right\|+\left\|\left|y_{\beta}-y\right| \cdot|x| \cdot u\right\|+\left\|\left|x_{\alpha}-x\right| \cdot|y| \cdot u\right\| .
\end{aligned}
$$

The second and the third terms in the last inequality both order converge to zero as $\beta \rightarrow \infty$ and $\alpha \rightarrow \infty$ respectively because of $|x| \cdot u,|y| \cdot u \in E_{+}$and $x_{\alpha} \stackrel{\mathrm{mn}}{\longrightarrow} x$ and $y_{\beta} \stackrel{\mathrm{mn}}{\longrightarrow} y$. Now, let's show the $m n$-convergence of the first term of last inequality. For fixed $u$, we can find two positive elements $u_{1}, u_{2} \in E_{+}$such that $u=u_{1} \cdot u_{2}$ because the positive element of $E$ can be written as a multiplication of two positive elements. So, we can get

$\left\|\left|x_{\alpha}-x\right| \cdot\left|y_{\beta}-y\right| \cdot u\right\|=\left\|\left(\left|x_{\alpha}-x\right| \cdot u_{1}\right) \cdot\left(\left|y_{\beta}-y\right| \cdot u_{2}\right)\right\| \leq\left\|\left|x_{\alpha}-x\right| \cdot u_{1}\right\| \cdot\left\|\left|y_{\beta}-y\right| \cdot u_{2}\right\|$.

Therefore, we see $\left|x_{\alpha}-x\right| \cdot\left|y_{\beta}-y\right| \cdot u \stackrel{\|\cdot\|_{0}}{\longrightarrow} 0$. Hence, we get $x_{\alpha} \cdot y_{\beta} \stackrel{\mathrm{mn}}{\longrightarrow} x \cdot y$.

In Theorem 2.10, the case of each positive element of $E$ can be written as a multiplication of two positive elements is called the factorization property for $f$-algebras in [13, Def.12.10]. But, instead of that property, we can give another easy condition in the following result.

Corollary 2.11. Let $E$ be a normed l-algebra, and $\left(x_{\alpha}\right)_{\alpha \in A}$ and $\left(y_{\beta}\right)_{\beta \in B}$ be two nets in $E$. If $x_{\alpha} \stackrel{\mathrm{mn}}{\longrightarrow} x$ and $y_{\beta} \stackrel{\mathrm{mn}}{\longrightarrow} y$ for some $x, y \in E$ and at least one of two nets is eventually norm bounded then we have $x_{\alpha} \cdot y_{\beta} \stackrel{\mathrm{mn}}{\longrightarrow} x \cdot y$.

Proof. Modify Theorem 2.10.

We give some basic notions motivated by their analogies from vector lattice theory.

Definition 2.12. Let $\left(x_{\alpha}\right)_{\alpha \in A}$ be a net in a normed $l$-algebra $E$. Then

(1) $\left(x_{\alpha}\right)$ is said to be $m n$-Cauchy if the net $\left(x_{\alpha}-x_{\alpha^{\prime}}\right)_{\left(\alpha, \alpha^{\prime}\right) \in A \times A} m n$-converges to 0 ,

(2) $E$ is called $m n$-complete if every $m n$-Cauchy net in $E$ is $m n$-convergent,

(3) $E$ is called $m n$-continuous if $x_{\alpha} \stackrel{\mathrm{o}}{\rightarrow} 0$ implies that $x_{\alpha} \stackrel{\mathrm{mn}}{\longrightarrow} 0$,

Proposition 2.13. A normed l-algebra is mn-continuous if and only if $x_{\alpha} \downarrow 0$ implies $x_{\alpha} \stackrel{\mathrm{mn}}{\longrightarrow} 0$.

Proof. Suppose any decreasing to zero net is $m n$-convergent to zero. We show $m n$ continuity. Let $\left(x_{\alpha}\right)_{\alpha \in A}$ be an order convergent to zero net in a normed $l$-algebra $E$. Then there exists another net $z_{\beta} \downarrow 0$ in $E$ such that, for any $\beta$ there exists $\alpha_{\beta}$ so that $\left|x_{\alpha}\right| \leq z_{\beta}$, and so $\left\|x_{\alpha}\right\| \leq\left\|z_{\beta}\right\|$ for all $\alpha \geq \alpha_{\beta}$. Since $z_{\beta} \downarrow 0$, by assumption, we have $z_{\beta} \stackrel{\mathrm{mn}}{\longrightarrow} 0$, i.e., for fixed $\varepsilon>0$ and $u \in E_{+}$, there is $\beta_{0}$ such that $\left\|z_{\beta} \cdot u\right\|<\varepsilon$ for all $\beta \geq \beta_{0}$. Thus, there exists an index $\alpha_{\beta_{0}}$ so that $\left\|\left|x_{\alpha}\right| \cdot u\right\| \leq \varepsilon$ for all $\alpha \geq \alpha_{\beta_{0}}$. Hence, $x_{\alpha} \stackrel{\mathrm{mn}}{\longrightarrow} 0$. The other case is obvious.

Proposition 2.14. Let $E$ be an mn-continuous and mn-complete normed l-algebra. Then every l-almost order bounded and order Cauchy net is mn-convergent. 
Proof. Let $\left(x_{\alpha}\right)_{\alpha \in A}$ be an l-almost order bounded order Cauchy net. Then the net $\left(x_{\alpha}-\right.$ $\left.x_{\alpha^{\prime}}\right)_{\left(\alpha, \alpha^{\prime}\right) \in A \times A}$ is $l$-almost order bounded and is order convergent to zero. Since $E$ is $m n$ continuous, $x_{\alpha}-x_{\alpha^{\prime}} \stackrel{\mathrm{mn}}{\longrightarrow} 0$. By using Proposition 2.8, we have $x_{\alpha}-x_{\alpha^{\prime}} \stackrel{\|\cdot\|_{0}}{\longrightarrow} 0$. Hence, we get that $\left(x_{\alpha}\right)_{\alpha \in A}$ is $m n$-Cauchy, and so it is $m n$-convergent because of $m n$-completeness.

\section{The $m n$-topology on normed $l$-algebra}

In this section, we now turn our attention to topology on normed $l$-algebras. We show that the $m n$-convergence in a normed $l$-algebra is topological. While mo- and uoconvergence need not be given by a topology. But, it was observed in [9] that the unconvergence is topological. Motivated from that definition of the $m n$-convergence, we give the following construction of the $m n$-topology.

Let $\varepsilon>0$ be given. For a non-zero positive vector $u \in E_{+}$, we put

$$
V_{u, \varepsilon}=\{x \in E:\||x| \cdot u\|<\varepsilon\} .
$$

Let $\mathcal{N}$ be the collection of all the sets of this form. We claim that $\mathcal{N}$ is a base of neighborhoods of zero for some Hausdorff linear topology. It is obvious that $x_{\alpha} \stackrel{\mathrm{mn}}{\longrightarrow} 0$ if and only if every set of $\mathcal{N}$ contains a tail of this net, hence the $m n$-convergence is the convergence induced by the mentioned topology.

We have to show that $\mathcal{N}$ is a base of neighborhoods of zero. To show this we apply [14, Thm.3.1.10.]. First, note that every element in $\mathcal{N}$ contains zero. Now, we show that for every two elements of $\mathcal{N}$, their intersection is again in $\mathcal{N}$. Take any two set $V_{u_{1}, \varepsilon_{1}}$ and $V_{u_{2}, \varepsilon_{2}}$ in $\mathcal{N}$. Put $\varepsilon=\varepsilon_{1} \wedge \varepsilon_{2}$ and $u=u_{1} \vee u_{2}$. We show that $V_{u, \varepsilon} \subseteq V_{u_{1}, \varepsilon_{1}} \cap V_{u_{2}, \varepsilon_{2}}$. For any $x \in V_{u, \varepsilon}$, we have $\||x| \cdot u\|<\varepsilon$. Thus, it follows from $|x| \cdot u_{1} \leq|x| \cdot u$ that

$$
\left\||x| \cdot u_{1}\right\| \leq\||x| \cdot u\|<\varepsilon \leq \varepsilon_{1} .
$$

Thus, we get $x \in V_{u_{1}, \varepsilon_{1}}$. By a similar way, we also have $x \in V_{u_{2}, \varepsilon_{2}}$.

Next, it is not a hard job to see that $V_{u, \varepsilon}+V_{u, \varepsilon} \subseteq V_{u, 2 \varepsilon}$, so that for each $U \in \mathcal{N}$, there is another $V \in \mathcal{N}$ such that $V+V \subseteq U$. In addition, one can easily verify that, for every $U \in \mathcal{N}$ and every scalar $\lambda$ with $|\lambda| \leq 1$, we have $\lambda U \subseteq U$.

Now, we show that, for each $U \in \mathcal{N}$ and each $y \in U$, there exists $V \in \mathcal{N}$ with $y+V \subseteq U$. Suppose $y \in V_{u, \varepsilon}$. We should find $\delta>0$ and a non-zero $v \in E_{+}$such that $y+V_{v, \delta} \subseteq V_{u, \varepsilon}$. Take $v:=u$. Hence, since $y \in V_{u, \varepsilon}$, we have $\||y| \cdot u\|<\varepsilon$. Put $\delta:=\varepsilon-\||y| \cdot u\|$. We claim that $y+V_{v, \delta} \subseteq V_{u, \varepsilon}$. Let's take $x \in V_{v, \delta}$. We show that $y+x \in V_{u, \varepsilon}$. Consider the inequality $|y+x| \cdot u \leq|y| \cdot u+|x| \cdot u$. Then we have

$$
\||y+x| \cdot u\| \leq\||y| \cdot u\|+\||x| \cdot u\|<\||y| \cdot u\|+\delta=\varepsilon .
$$

Finally, we show that this topology is Hausdorff. It is enough to show that $\bigcap \mathcal{N}=\{0\}$. Suppose that it is not hold true, i.e., assume that $0 \neq x \in V_{u, \varepsilon}$ for all non-zero $u \in E_{+}$and for all $\varepsilon>0$. In particular, take $x \in V_{|x|, \varepsilon}$. Thus, we have $\left\||x|^{2}\right\|<\varepsilon$. Since $\varepsilon$ is arbitrary, we get $|x|^{2}=0$, i.e., $x=0$ by using [17, Thm.142.3.]; a contradiction.

Recall that the statement $V_{u, \varepsilon}$ is either contained in $[-u, u]$ or contains a non-trivial ideal holds true for the un-topology. However, it is not true for the $m n$-topology. To see this, we give the following counterexample.

Example 3.1. Consider the $l$-algebra $E=C[0,1]$ with the sup-norm topology $\tau$. Take $a=\mathbb{1}$ and $A=B(0,10)$. The set $U_{a, A}=\{x \in E:|x| \cdot a \in A\}=B(0,10)$ is neither contained in $[-a, a]=[-\mathbb{1}, \mathbb{1}]=B(0,1)$ nor contains a non-trivial ideal. 
Lemma 3.2. If $V_{u, \varepsilon}$ is contained in $[-u, u]$, then $u$ is a strong unit.

Proof. Take a positive element $x \in E_{+}$. Then we have a positive scalar $\lambda$ such that $(\lambda x) \cdot a \in A$. Thus we get $\lambda x \in U_{a, A}$ and so, $\lambda x \in[-a, a]$. Then one can see that $a$ is a strong unit.

\section{The $m n$-convergence on semiprime normed $f$-algebras}

Recall that an element $x$ in an $f$-algebra $E$ is called nilpotent whenever $x^{n}=0$ for some natural number $n \in \mathbb{N}$. The algebra $E$ is called semiprime if the only nilpotent element in $E$ is the null element ([17, p.670]). We begin the section with the next useful result.

Proposition 4.1. Let $\left(x_{\alpha}\right)_{\alpha \in A}$ be a net in nilpotent elements of a normed f-algebra $E$. If $x_{\alpha} \stackrel{\mathrm{mn}}{\longrightarrow} x$ then $x$ is also a nilpotent element.

Proof. Take a fixed positive element $u \in E_{+}$. Then, by using [13, Prop.10.2(iii)] and [17, Thm.142.1(ii)], we get

$$
\left\|\left|x_{\alpha}-x\right| \cdot u\right\|=\left\|\left|x_{\alpha} \cdot u-x \cdot u\right|\right\|=\left\|x_{\alpha} \cdot u-x \cdot u\right\|=\|x \cdot u\| \rightarrow 0 .
$$

Thus $\|x \cdot u\|=0$ and hence $x \cdot u=0$ for every $u \in X_{+}$. Then $y \cdot x=0$ for all $y \in E$. It follows now from $[12$, p.157] that $x$ is nilpotent in $E$.

Remark 4.2. By considering Proposition 4.1, it is easy to see that $m n$-convergence in normed $f$-algebra $E$ has an unique limit if and only if $E$ is semiprime normed $f$-algebra.

Unless stated otherwise, we will assume that $E$ is a semiprime normed $f$-algebra and all nets and vectors lie in $E$.

Proposition 4.3. Let $\left(x_{\alpha}\right)_{\alpha \in A}$ be a net in $E$. Then we have that

(i) $0 \leq x_{\alpha} \stackrel{\mathrm{mn}}{\longrightarrow} x$ implies $x \in E_{+}$,

(ii) if $\left(x_{\alpha}\right)$ is monotone and $x_{\alpha} \stackrel{\mathrm{mn}}{\longrightarrow} x$ then $x_{\alpha} \stackrel{\mathrm{o}}{\rightarrow} x$.

Proof. (i) Assume $\left(x_{\alpha}\right)_{\alpha \in A}$ consists of non-zero elements and $m n$-converges to $x \in E$. Then, by using Proposition 2.2, we have $x_{\alpha}=x_{\alpha}^{+} \stackrel{\mathrm{mn}}{\longrightarrow} x^{+}$. Also, following from Remark 4.2 , we get $x^{+}=x$. Therefore, we get $x \in E_{+}$.

(ii) For the order convergence of $\left(x_{\alpha}\right)_{\alpha \in A}$, it is enough to show that $x_{\alpha} \uparrow$ and $x_{\alpha} \stackrel{\mathrm{mn}}{\longrightarrow} x$ implies $x_{\alpha} \stackrel{\circ}{\rightarrow} x$. For a fixed index $\alpha$, we have $x_{\beta}-x_{\alpha} \in X_{+}$for all $\beta \geq \alpha$. By applying (i), we can see $x_{\beta}-x_{\alpha} \stackrel{\mathrm{mn}}{\longrightarrow} x-x_{\alpha} \in X_{+}$as $\beta \rightarrow \infty$. Therefore, $x \geq x_{\alpha}$ for the index $\alpha$. Since $\alpha$ is arbitrary, $x$ is an upper bound of $\left(x_{\alpha}\right)$. Assume $y$ is another upper bound of $\left(x_{\alpha}\right)$, i.e., $y \geq x_{\alpha}$ for all $\alpha$. So, $y-x_{\alpha} \stackrel{\mathrm{mn}}{\longrightarrow} y-x \in X_{+}$, or $y \geq x$, and so $x_{\alpha} \uparrow x$.

Theorem 4.4. The following statements are equivalent:

(i) $E$ is mn-continuous;

(ii) if $0 \leq x_{\alpha} \uparrow \leq x$ holds in $E$ then $\left(x_{\alpha}\right)$ is an mn-Cauchy net;

(iii) $x_{\alpha} \downarrow 0$ implies $x_{\alpha} \stackrel{\mathrm{mn}}{\longrightarrow} 0$ in $E$.

Proof. (i) $\Rightarrow$ (ii) Take a net $0 \leq x_{\alpha} \uparrow \leq x$ in $E$. Then there exists another net $\left(y_{\beta}\right)$ in $E$ such that $\left(y_{\beta}-x_{\alpha}\right)_{\alpha, \beta} \downarrow 0$; see [2, Lem.4.8]. Thus, by applying Proposition 2.13, we have $\left(y_{\beta}-x_{\alpha}\right)_{\alpha, \beta} \stackrel{\mathrm{mn}}{\longrightarrow} 0$ because $E$ is $m n$-continuous. Therefore, the net $\left(x_{\alpha}\right)$ is $m n$-Cauchy because of $\left\|x_{\alpha}-x_{\alpha^{\prime}}\right\|_{\alpha, \alpha^{\prime} \in A} \leq\left\|x_{\alpha}-y_{\beta}\right\|+\left\|y_{\beta}-x_{\alpha^{\prime}}\right\|$.

(ii) $\Rightarrow$ (iii) Put $x_{\alpha} \downarrow 0$ in $E$ and fix arbitrary $\alpha_{0}$. Thus, we have $x_{\alpha} \leq x_{\alpha_{0}}$ for all $\alpha \geq \alpha_{0}$, and so we can get $0 \leq\left(x_{\alpha_{0}}-x_{\alpha}\right)_{\alpha \geq \alpha_{0}} \uparrow \leq x_{\alpha_{0}}$. Then it follows from (ii) that the net $\left(x_{\alpha_{0}}-x_{\alpha}\right)_{\alpha \geq \alpha_{0}}$ is $m n$-Cauchy, i.e., $\left(x_{\alpha^{\prime}}-x_{\alpha}\right) \stackrel{\mathrm{mn}}{\longrightarrow} 0$ as $\alpha_{0} \leq \alpha, \alpha^{\prime} \rightarrow \infty$. Since $E$ is $m n$ complete, there exists an element $x \in E$ satisfying $x_{\alpha} \stackrel{\mathrm{mo}}{\longrightarrow} x$ as $\alpha_{0} \leq \alpha \rightarrow \infty$. It follows 
from Proposition 4.3 that $x_{\alpha} \downarrow 0$ because of $x_{\alpha} \downarrow$ and $x_{\alpha} \stackrel{\mathrm{mn}}{\longrightarrow} 0$, and so, following from Remark 4.2 tha we have $x=0$. Therefore, we get $x_{\alpha} \stackrel{\mathrm{mn}}{\longrightarrow} 0$.

(iii) $\Rightarrow$ (i) It is just the implication of Proposition 2.13.

Corollary 4.5. Every mn-continuous and $m n$-complete normed $f$-algebra $E$ is order complete.

Proof. Suppose $E$ is $m n$-continuous and $m n$-complete. For $y \in E_{+}$, put a net $0 \leq x_{\alpha} \uparrow \leq y$ in E. By applying Theorem $4.4(i i)$, the net $\left(x_{\alpha}\right)$ is $m n$-Cauchy. Thus, there exists an element $x \in E$ such that $x_{\alpha} \stackrel{\mathrm{mn}}{\longrightarrow} x$ because of $m n$-completeness. Since $x_{\alpha} \uparrow$ and $x_{\alpha} \stackrel{\mathrm{mo}}{\longrightarrow} x$, it follows from Lemma 4.3 that $x_{\alpha} \uparrow x$. Therefore, $E$ is order complete.

Acknowledgment. The author would like to thank Eduard Emelyanov and Mohamed Ali Toumi for improving the paper.

\section{References}

[1] Y. Abramovich and C.D. Aliprantis, An Invitation to Operator Theory, American Mathematical Society, New York, 2003.

[2] C.D. Aliprantis and O. Burkinshaw, Positive Operators, Springer, Dordrecht, 2006.

[3] A. Aydın, Unbounded $p_{\tau}$-convergence in vector lattice normed by locally solid vector lattices, in: Academic Studies in Mathematics and Natural Sciences-2019/2, 118-134, IVPE, Cetinje-Montenegro, 2019.

[4] A. Aydn, Multiplicative order convergence in f-algebras, Hacet. J. Math. Stat. 49 (3), 998-1005, 2020.

[5] A. Aydın, E. Emel'yanov, N.E. Özcan, and M.A.A. Marabeh, Compact-like operators in lattice-normed spaces, Indag. Math. 2, 633-656, 2018.

[6] A. Aydın, E. Emel'yanov, N.E. Özcan, and M.A.A. Marabeh, Unbounded pconvergence in lattice-normed vector lattices, Sib. Adv. Math. 29, 153-181, 2019.

[7] A. Aydın, S.G. Gorokhova, and H. Gül, Nonstandard hulls of lattice-normed ordered vector spaces, Turkish J. Math. 42, 155-163, 2018.

[8] Y.A. Dabboorasad, E.Y. Emelyanov, and M.A.A. Marabeh, $u \tau$-Convergence in locally solid vector lattices, Positivity 22, 1065-1080, 2018.

[9] Y. Deng, M. O'Brien, and V.G. Troitsky, Unbounded norm convergence in Banach lattices, Positivity 21, 963-974, 2017.

[10] N. Gao, V.G. Troitsky, and F. Xanthos, Uo-convergence and its applications to Cesáro means in Banach lattices, Israel J. Math. 220, 649-689, 2017.

[11] N. Gao and F. Xanthos, Unbounded order convergence and application to martingales without probability, Math. Anal. Appl. 415, 931-947, 2014.

[12] C.B. Huijsmans and B.D. Pagter, Ideal theory in $f$-algebras, Trans. Amer. Math. Soc. 269, 225-245, 1982.

[13] B.D. Pagter, f-Algebras and Orthomorphisms, Ph.D. Dissertation, Leiden, 1981.

[14] V. Runde, A Taste of Topology, Springer, Berlin, 2005.

[15] V.G. Troitsky, Measures of non-compactness of operators on Banach lattices, Positivity 8, 165-178, 2004.

[16] B.Z. Vulikh, Introduction to the Theory of Partially Ordered Spaces, WoltersNoordhoff Scientific Publications, Groningen, 1967.

[17] A.C. Zaanen, Riesz Spaces II, The Netherlands: North-Holland Publishing Co., Amsterdam, 1983. 